\title{
Research on Pathological Mechanism of Depression and Antidepressant Drug Target
}

\author{
Yu Liü, a, Bin $\mathrm{Wu}^{1}$, Liguo Guo ${ }^{1}$, Yongzhi Li ${ }^{1}$ \\ ${ }^{1}$ China Astronaut Research And Training Center , Beijing, 100094, China
}

Keywords: Depression, Antidepressant drugs, Drug target

\begin{abstract}
Depression is a common disease that endangers people's health. The etiology of depression is more complex, and the possible reasons include low immunologic function, neuroendocrine functional disorder and astrocyte cell dysfunction. In this paper, we investigate the role of monoamine oxidase inhibitors, 5-HT reuptake inhibitors and tricyclic antidepressants to provide some references for the relative researchers.
\end{abstract}

\section{Introduction}

Depression is a common mental disease, characterized by a long period of depression, loss of interest and happiness, and can lead to serious cardiovascular and cerebrovascular diseases. Currently, depression has become a worldwide disease. It is estimated that by 2020, the global incidence of depression will rank $2^{\text {nd }}$, only below the coronary heart disease, ranking second. The pathogenesis of depression is a complex process involving multiple systems and links. Depression is usually referred to as an emotional disorder, a syndrome characterized by low mood. The main clinical manifestations of emotion, interest, perception, thinking, volition and low physiological function disorder, even stupor, or hallucinations, delusions and other psychotic symptoms. Some patients with suicide, or accompanied by significant anxiety and irritability, and even attack behavior. Depressive episodes were divided into single episode depression and recurrent depression! At present, there is no psychotic symptom of depression and depression is currently a psychotic symptoms. Light depression refers to the diagnostic criteria of depression, but the social function no damage or only mild damage; recurrent depression refers to the attack in accordance with the diagnostic criteria of depression, and in the interval of at least two months ago had another attack, but there has never been a mania with any mania, bipolar disorder. Ring or affective disorder; and to exclude organic mental disorders, or psychoactive substances and non-addictive substance induced depression. The psychotic symptoms refer to the hallucinations, delusions, or tension syndrome.

\section{Pathological Mechanism of Depression}

Hypothesis of Low Immunologic Function. Immunologic function refers to the body's resistance to disease, immune function is accomplished in lymphocytes, monocytes and other cells and their products under the interaction of the immune system; immune function play a role based on immune recognition. The activation of inflammatory mediators of depression and possible mechanism of cytokines and other immune system, they can cause changes in behavior, such as anorexia, lethargy, loss of body mass and activity decreased. Slow injection of LPS in healthy volunteers, in which activation and related anxiety, depressive mood and memory impairment caused by immune positive individual physiological diseases, the level of cytokines mood disorders and cognitive impairment and LPS induced. Animal experiments also found that animal autoimmune disease is often accompanied by saccharin reward response reduction. These studies suggest that cytokines may be involved in the occurrence of depression in patients with immune activation related diseases. At the same time, depression often occurs in many chronic activation of the immune system related non infectious diseases, such as multiple sclerosis, allergy, rheumatoid arthritis and stroke, and anti-inflammatory cytokine therapy can significantly improve the patient's symptoms of depression. These diseases are associated with depression like symptoms that may not be due to physiological 
illness, such as pain, depression and other psychological reactions, but directly by the immune activation and cytokine release. Studies have shown that immune disorders occur prior to depression, and the use of cytokine inhibitors in advance can alleviate these symptoms associated with immune activation.

Hypothesis of Neuroendocrine Functional Disorder. It is found that HPA is involved in the pathological mechanism of depression. Studies show that it plays an important role in the pathogenesis of depression, plays a key role in the regulation of stress response, and some depression symptoms and signs in the common pathway. Patients with depression often appear HPA axis hyper function, resulting in excessive secretion of cortisol, the secretion of circadian rhythm has also changed, and then damage the hippocampus neurons in patients with brain, leading to cognitive decline. The main function of HPA axis in patients with depression is the enhancement of secretion and the slow release of thyrotrophic releasing hormone. It has been found that the damage of axis is more severe in patients with refractory depression. In addition, the phenomenon of axial activation in the plasma of rats with chronic stress often appears as a marked increase. The axis play a role in the activation step is first through the hypothalamic pituitary portal system the secretion of hypothalamic regulatory peptide delivered to the pituitary to regulate the pituitary gland, then in the regulation of peptide under the action of pituitary release, the final effect on the adrenal cortex through the release of adrenal cortex hormones into the body. The incidence of depression is related to the apoptosis of hippocampal neurons, the reduction of hippocampal volume and the loss of some neurons. It has been found that acute or chronic stress stimulation can decrease the expression of hippocampus.

Hypothesis of Astrocyte Cell Dysfunction. Astrocytes are the most widely distributed cells in the brain of mammals, and are one of the largest cells in glial cells. Metal dip technology classic shows that such star shaped glial cells, make a lot of long and branching projections from the cell body, stretching between nerve cells filled in the cell body and processes, supporting and separating the nerve cells. Some feet attached to the capillary wall on the adjacent. The board is also known as vascular foot or perivascular feet, close to the surface of the brain and spinal cord is attached on the surface of the soft baseboard, connected to each other constitute glia. Astrocytes in the central nervous system, the largest number of the most widely distributed, can secrete various neurotropic factors such as BNDF, nerve growth factor, glial cell line derived neurotropic factor; nerve can secrete various neurotropic factors such as support membrane binding molecules, cell adhesion molecules. Astrocyte as the neuron supporting cells, with the role of nutritional support on neurons, can induce cell neurogenesis and synaptic remodeling, nerve stem, is also responsible for mediating excitatory synaptic transmission and glutamate nerve excitability increased due to the cleaning in the synaptic gap. From the above knowledge, stress will make the glutamic acid content in brain and the abnormal increase of extracellular glutamate amino acid transporter astrocyte on scavenging. The fluoxetine can improve depression psychomotor depression and weakness, fatigue, can be effective in the treatment of bulimia nervosa and binge eating episodes, does not cause weight gain, but should be violent and prevent the phenomenon after medication: Pa Rossi Dean is better for patients with anxiety and depression, but some patients because of taking the drug caused by weight increased sexual dysfunction. Seculin is particularly suitable for women and the elderly depression. Fluvoxamine is considered to be the best drug in the treatment of obsessive-compulsive disorder SSRI, citalopram and other drugs interaction at least, especially suitable for a variety of drugs need to be together. If astrocyte dysfunction, brain glutamate cannot be cleared in time, will lead to depression. Therefore, the production of depression is related to the dysfunction of astrocyte. The expression of glial acidic protein in astrocyte plays an important role.

\section{Antidepressant Drug Target}

Monoamine Oxidase Inhibitors. Monoamine oxidase is the deamination of catalytic oxidation of the enzyme monoamine substance. It exists in the outer membrane of mitochondria, and is widely distributed in the human body. The main physiological function of the disease is to regulate the concentration of the corresponding amine in the organism through the oxidation reaction, so that the 
primary amine and its methylated two or three - level amine or the long chain of the two amino acid inactivation. Monoamine oxidase inhibitors are drugs clinically by inhibiting the oxidative monoamine substance exerts its pharmacological action. It can be divided into reversible and irreversible inhibitors, and can be divided into selective and non-selective inhibitors. Different pharmacological effects are also different. In clinical application, monoamine oxidase inhibitors are widely used. The early treatment of depression of the first generation of antidepressants is irreversible MAO inhibitor, which was first used in the treatment of tuberculosis in 1950s found that the excitement of the central role, and subsequently used in the treatment of depressive psychosis. The mechanism is that nerve cells in the brain MAO activity decreased, resulting in decreased degradation of monoamine neurotransmitters, so as to improve the content of NE, 5-HT and DA play a role of anti-depression. Belong to this kind of medicaments still have benzene to wait. Compared with tricyclic antidepressants, these drugs are effective in the treatment of severe depression (major depression disorder). Although they have strong endogenous excitatory effect, slow action and durable, but also have serious side effects, easy to cause liver injury and severe hypertensive crisis. Therefore, it is quickly replaced by the second generation of tricyclic antidepressants in the clinic. It overcomes the shortcomings of the old generation of liver toxicity and hypertensive crisis, at the same time has not increased weight, does not affect the advantages of sexual function and cognitive function, safety and better than tricyclic antidepressants. The efficacy of MDD was similar to that of selective five light amine reuptake inhibitors, but was better in patients with tolerance. Studies have shown that it has a more significant effect on atypical depression and post stroke depression than other types of antidepressants.

5-HT Reuptake Inhibitors. The pathogenesis of depression and the evaluation of antidepressant drugs are all dependent on the animal model of depression. The core symptoms and pathophysiological changes of depression depend on the complex structure of the brain, while the current animal model is mainly used in normal animals, and can only partially simulate the occurrence of depression. Therefore, most animal models of depression have some limitations. However, the commonly used animal models of depression still provide important evidence that 5-HT receptor is associated with depression. It was found that the overexpression of 5-HT receptor $30 \%$ could decrease the activity of 5-HT neurons, leading to the decrease of 5-HT release and the increase of depression in rats. Therefore, the increased expression of 5-HT receptor and the inhibition of 5-HT neurotransmission are the main causes of depression. In addition, 5-HT receptor knockout mice, due to the lack of inhibition of presynaptic 5-HT receptor immediately increase the release of 5-HT. But at the same time due to lack of postsynaptic 5-HT receptors, fluoxetine does not show obvious antidepressant effect, suggesting that the 5-HT receptor is associated with depression, and the presynaptic and postsynaptic 5-HT receptors in antidepressant therapy plays an important role. Negative feedback inhibition caused by 5-HT receptor activation plays a key role in the onset of antidepressant drugs. Therefore, if we can develop antidepressant drugs with 5-HT reuptake inhibition and presynaptic 5-HT receptor antagonist can release the presynaptic activation of 5-HT receptor negative feedback inhibition caused, achieve the purpose of rapid onset of action. Therefore, the design and synthesis of both 5-HT receptor agonist and 5-HT reuptake inhibition of the dual role of antidepressant drugs not only acts on the presynaptic 5-HT receptor, promote its desensitization. At the same time, it can directly stimulate the postsynaptic membrane 5-HT receptor, promote 5-HT nerve function. It may have a strong and rapid antidepressant effect. This vision is expected to represent the direction of research and development of new antidepressants.

Tricyclic Inhibitors. Tricyclic antidepressants are commonly used amitriptyline and doxepin, imipramine equal. The mechanism of TCA is usually thought to block the reuptake of norepinephrine and 5-HT in the presynaptic membrane of the nerve terminals, and increase the concentration of monoamine neurotransmitters in the synaptic gap. TCA is fat soluble, good oral absorption, plasma protein binding rate of $90 \%-95 \%$, widely distributed in various tissues of the body. TCA is metabolized in the liver, and many of its metabolites also have biological activity. But its plasma half-life is longer, it takes L-3 weeks to reach steady state plasma concentration. TCA inhibited the acetylcholine receptor, histamine receptor and norepinephrine receptors, so prone to adverse 
reactions during the treatment. A series of tricyclic drug rings and have developed into imipramine and tetracycline drug has well anti depression effect, become the main drug in the treatment of depression. In the first generation of monoamine reuptake inhibitors, it not only inhibits the reuptake of light amine and norepinephrine, but also has anticholinergic effects. The adverse reactions of these drugs mainly from anticholinergic effects, such as dry mouth, constipation, urinary retention, blurred vision and eye rise higher, is the most serious cardiac toxicity, especially in elderly patients are more likely to happen, such as orthostatic hypotension, arrhythmia, block, heart failure, myocardial infarction, etc.. The safety of low toxic concentration is only ten times the treatment concentration, so it has a narrow therapeutic window, after poisoning can lead to fatal arrhythmia, must be regular monitoring of blood drug concentration, which will increase the cost of treatment, reduce compliance. Therefore, it is generally used only in patients with major depression or invalid SSRI class. This kind of medicine is developed based on tricyclic antidepressants on, its purpose is to increase the efficacy and reduce adverse reactions. The dosage for Planck forest was 75-150 mg daily, the daily maximum dose should not exceed $200 \mathrm{mg}$. The drug effect of anti-depression and amitriptyline also has the same. Obvious sedative and anxiolytic effects, but the drug induced epilepsy. Side effects of tetracyclic antidepressants and tricyclic antidepressants are similar, but compared to tricyclic antidepressants. The reaction of the drug side effect is lighter.

\section{Conclusion}

Depression is a serious mental disorder which can harm human health severely. Currently, the most common treatment is the use of drugs such as monoamine oxidase inhibitors, 5-HT reuptake inhibitors and tricyclic inhibitors. However, these drugs have the disadvantages of slow treatment effectiveness, long-term medication and high recurrence rate. It is the focus of future research on depression treatment to obtain more effective treatments and develop the efficient antidepressant drugs.

\section{Acknowledgements}

This research is the result of Major Project (Grant No. AWS16J018) supported by the Foundation item1226.

\section{References}

[1] Chen Hongxia, Zhang Liming, Xue Rui, Li Yunfeng, Progress of antidepressant drugs based on 5- $\mathrm{HT}_{1 \mathrm{~A}}$ receptor as a new target [J]. Military Medical Sciences, 2013,37 (8): 624-627.

[2] Che Xiaohang, Ye Xiaolei, Chen Chunlin. Progress of non-monoaminergic targets for antidepressant therapies [J]. Chinese Journal of Clinical Pharmacology and Therapeutics, 2015, 20(8): 943-949.

[3] Mu Lei, Sun Jianxu. Regulation mechanism of depression and antidepressant drug targets [J]. Journal of International Pharmaceutical Research, 2015, 42(4): 463-466.

[4] Gao Yao, Gao Li, Gao Xiaoxia, Zhou Yuzhi, Qin Xuemei, Tian Junsheng. An exploration in the action targets for antidepressant bioactive components of Xiaoyaosan based on network pharmacology [J]. Acta Pharmaceutica Sinica, 2015, 50 (12): 1589-1595. 